\title{
Atuação do enfermeiro frente a qualidade de vida de mulheres climatéricas com incontinência urinária: revisão integrativa da literatura
}

Nurses' performance in relation to the quality of life of climacteric women with urinary

incontinence: integrative literature review

Actuación del enfermero en relación a la calidad de vida de mujeres climatéricas con incontinencia urinaria: revisión integrativa de la literatura

Recebido: 03/02/2022 | Revisado: 08/02/2022 | Aceito: 16/02/2022 | Publicado: 23/02/2022

Ângela Roberta Lessa de Andrade
ORCID: https://orcid.org/0000-0001-7753-675X
Universidade de Pernambuco, Brasil
E-mail: angelalessadeandrade @ yahoo.com.br
Alice Fonseca Pontes
ORCID: https://orcid.org/0000-0002-3291-5964
Universidade de Pernambuco, Brasil
E-mail: alicepontes136@ gmail.com
Jabiael Carneiro da Silva Filho
ORCID: https://orcid.org/0000-0002-1609-1125
Universidade de Pernambuco, Brasil
E-mail: jabiaelfilho@gmail.com
Isabel Cristina Ramos Vieira Santos
ORCID: https://orcid.org/0000-0002-5458-4334
Universidade de Pernambuco, Brasil
E-mail: isabel.santos@ upe.br

\section{Resumo}

Objetivou-se a identificação da IU como fator associado à diminuição da QV em mulheres climatéricas, e tornou-se relevante subsidiar as estratégias que visem à promoção da saúde, o alívio dos sintomas, à prevenção e controle da IU. E desta forma, melhorar a QV destas mulheres com uma abordagem inclusiva, holística e humanizada. A amostra final desta revisão foi formada por nove artigos datados entre os anos de 1998 e 2021. Na seleção dos materiais utilizados encontram-se os resultados obtidos na caracterização da produção científica sobre as formas de avaliação da força da musculatura do assoalho pélvico e técnicas de fortalecimento deste, em mulheres com IU. A atual revisão demonstrou que as publicações realizadas nesta área, e principalmente por Enfermeiros, ainda tem pouca representatividade, e que devido à relevância do tema precisamos desenvolver outros estudos evidenciando a relevância do trabalho do enfermeiro, no ato da consulta de enfermagem e seu amparo legal, avaliando a força dos músculos do assoalho pélvico, e se houver a detecção do enfraquecimento muscular iniciar a orientação sobre a importância do fortalecimento muscular na sustentação e da continência dos órgãos pélvicos, além de orientar que este fortalecimento. Orientar que deve ser uma atividade supervisionada com as devidas orientações durante as consultas e em grupos focais, de modo a melhorar a QV da população feminina de uma forma geral, com informações fáceis, que transmitem segurança às mulheres e incentivam este tratamento que permite manter a rotina e convívio social da mulher.

Palavras-chave: Incontinência Urinária; Enfermagem; Assoalho pélvico; Qualidade de vida.

\begin{abstract}
The objective was to identify UI as a factor associated with decreased QoL in climacteric women, and it became relevant to support strategies aimed at promoting health, relieving symptoms, preventing and controlling UI. And in this way, improve the QOL of these women with an inclusive, holistic and humanized approach. The final sample of this review consisted of nine articles dated between 1998 and 2021. In the selection of the materials used, the results obtained in the characterization of the scientific production on the forms of evaluation of the strength of the pelvic floor muscles and techniques of strengthening this, in women with UI. The current review showed that the publications carried out in this area, and especially by nurses, still have little representation, and that due to the relevance of the topic we need to develop other studies showing the relevance of the nurse's work, in the act of the nursing consultation and its legal support., evaluating the strength of the pelvic floor muscles, and if there is a detection of muscle weakness, start the guidance on the importance of muscle strengthening in the support and continence of Organs pelvic organs, in addition to guiding that this strengthening. Advise that it should be a supervised activity with the proper guidelines during consultations and in focus groups, in order to improve the QOL of the female population in general, with easy information, which convey security to women and encourage this treatment that allows maintaining the routine and
\end{abstract}


social life of women.

Keywords: Urinary incontinence; Nursing; Pelvic floor; Quality of life.

\begin{abstract}
Resumen
Objetivou-se a identificação da IU como fator associado à diminuição da QV em mulheres climatéricas, e tornou-se relevante subsidiar as estratégias que visem à promoção da saúde, o alívio dos sintomas, à prevenção e controle da IU. E desta forma, melhorar a QV destas mulheres com uma abordagem inclusiva, holística e humanizada. A mostra final desta revisão foi formado por nove artigos datados between os anos of 1998 and 2021. Na seleção dos materies used encontram-se os resultados obtidos na caracterização da produção cientific sobre as formes de avaliação da força da musculatura do assoalho pélvico e técnicas de fortalecimento deste, em mulheres com IU. A atual revisão demonstrou que as publicações made nesta area, e principalmente for Enfermeiros, ainda tem pouca representatividade, e que devido à relevância do tema precisamos desenvolver outros estudos evidenciando a relevância do trabalho do enfermeiro, no ato da consult of enfermagem and seu amparo legal , avaliando a força dos músculos do assoalho pélvico, y se houver a detecção do enfraquecimento muscular iniciar a orientação on a importância do fortalecimento muscular na sustentação e da continência dos órgãos pélvicos, além de orientar que este fortalecimento. Orientar que deve ser uma atividade supervisionada com as devidas orientações durante as consultas e em grupos focais, de modo a melhorar a QV da população feminina of uma form geral, com informações fáceis, que transmitm safença às mulheres e incentivam este tratamento que permite manter a Rotina y convívio social de mujer.

Palabras clave: Incontinencia urinaria; Enfermería; Suelo pélvico; Calidad de vida.
\end{abstract}

\title{
1. Introdução
}

A definição de climatério pela Organização Mundial de Saúde (OMS) é que se trata de uma fase biológica da vida e não um processo patológico, que compreende a transição entre o período reprodutivo e o não reprodutivo da vida da mulher, ocorrendo modificações hormonais e comportamentais (Brasil, 2013). A menopausa é um marco dessa fase, correspondendo ao último ciclo menstrual, somente reconhecida depois de passados 12 meses da sua ocorrência e acontece geralmente em torno dos 48 aos 50 anos de idade, estando relacionada à suspensão da foliculogênese ovariana (Brasil, 2016).

O Ministério da Saúde divide o climatério em três fases: a primeira (chamada de precoce) vai dos 35 aos 45 anos; a segunda fase (chamada de perimenopausa) vai dos 45 aos 55 anos; e a terceira (chamada de tardia) vai dos 55 aos 65 anos. A perimenopausa é dividida em duas fases: a pré e a pós-menopausa, sendo essa segunda fase a que possui maiores repercussões para o cotidiano feminino (Brasil, 2008).

A característica deste período são as mudanças endócrinas (devido ao declínio da atividade ovariana), mudanças biológicas (em função da diminuição da fertilidade) e mudanças clínicas (consequentes das alterações do ciclo menstrual) (Bertoldi, et al., 2014; Oliveira, et al., 2018). De acordo com Botelho, et al. (2007) os sinais e sintomas clínicos do climatério são divididos em transitórios e não transitórios. Os transitórios são representados pelas alterações do ciclo menstrual e pela sintomatologia mais aguda dos sintomas vasomotores, neuropsíquicos e as disfunções sexuais. Os não transitórios são representados pelos fenômenos atróficos geniturinários, distúrbios no metabolismo lipídico e ósseo (Brasil, 2008).

Focando nestes fenômenos geniturinários, destacamos a Incontinência Urinária (IU), que é definida pela International Continence Society, como qualquer perda involuntária de urina, e pode ser dividida em 3 tipos principais: urgência, esforço e mista (que tem características dos dois tipos) (Paris, 2008).

Sendo a IU é um problema de saúde pública e destaca a necessidade do olhar à saúde da mulher, por ser uma doença multifatorial, que acontece em qualquer idade, principalmente no climatério, e que acomete aproximadamente $27,6 \%$ das mulheres comparado à 10,5\% dos homens, refletindo nas relações interpessoais, profissionais e sexuais. Dá destaque aos achados da pesquisa que realizou onde descreve como fatores de risco: consumo de cafeína (97,9\%), cirurgia ginecológica (87,5\%), infecção urinária $(62,5 \%)$ e prática de atividade física $(56,3 \%)$. Acrescenta que após exposição a tais fatores, o tempo de início da doença foi entre um e cinco anos $(43,8 \%)$. Onde após este período as mulheres apresentaram episódios de perda de urina caracterizando IU de esforço, e estas mulheres desenvolveram isolamento social, déficit de autoestima e adoção de medidas não resolutivas como uso de absorventes. Apenas 33,3\% procuraram um médico, 35,4\% das que foram atendidas receberam 
orientações sobre a doença, apenas $25 \%$ conheceram o diagnóstico médico e desta forma 12,5\% realizaram algum tipo de tratamento (Mourão, et al., 2017).

Conforme literatura a IU repercute na qualidade de vida (QV), e desta forma, e demonstrando um problema que precisamos enfrentar (Guarisi, et al., 2001; Ferreira \& Santos, 2009). Entre várias formas de tratamento para a IU estudadas, o fortalecimento da musculatura do assoalho pélvico (FMAP) popularizou-se após os estudos de Arnold Kegel, na década de 1950, e até hoje estes exercícios são a base dos exercícios de FMAP, visto ser fácil aceitação pelas pacientes, serem efetivos e não invasivos, tratando-as de forma que muitas ficam curadas da IU e as outras referem melhora significativa (Sousa, Souza \& Figueredo, 2020). Através de um programa de treino de força, que incorpora um treinamento progressivo dos parâmetros: intensidade, frequência, tempo de repouso, volume, duração e especificidade do movimento.

A reabilitação da musculatura do assoalho pélvico com finalidade uroginecológica e obstétrica é legalmente atividade do enfermeiro, visto que não é um procedimento invasivo, e que faz parte da consulta de enfermagem individual e em equipe multiprofissional (COFEN, 2016). Em 2021, Santos e Menezes afirmaram que não há impeditivo legal para a prática da reabilitação pélvica pelo enfermeiro orientando a busca de conhecimento e habilidade na reabilitação do assoalho pélvico para que esta prática assistencial seja efetiva, e ainda ressalta que a atuação do enfermeiro melhora a QV dos pacientes.

Diante da complexidade da síndrome climatérica, destacando a IU, e os seus possíveis reflexos sobre a QV, fazemse necessário uma nova abordagem, destacando a importância dos aspectos psicológicos relacionados a este complexo processo fisiológico com interferência psicológica e social (SOBRAC, 2003). Desta forma, de fato, com o que se relacionada a QV de mulheres que passam pelo período do climatério? Portanto, a presente pesquisa teve por objetivo avaliar a QV de mulheres na fase do climatério.

Nesse contexto, objetivou-se a identificação da IU como fator associado à diminuição da QV em mulheres climatéricas tornou-se relevante subsidiar a estratégias que visem à promoção da saúde, o alívio dos sintomas, à prevenção e ao controle da IU, e assim, melhorar a QV destas mulheres com uma abordagem inclusiva, holística e humanizada.

\section{Metodologia}

Trata-se de uma revisão integrativa da literatura, com a finalidade de reunir resultados de investigações sobre o tema, conduzida no intuito de atender à questão norteadora: "Como melhorar a qualidade de vida de mulheres climatéricas com IU?".

Esse tipo de estudo nos facilita a integração de trabalhos experimentais e os não-experimentais, para que possa ser compreendido de forma integral o evento/fenômeno estudado (Souza, et al 2010). Tendo assim, sua finalidade a síntese dos resultados adquiridos em pesquisas sobre um determinado assunto almejado, de forma metódica e ordenada, fornecendo informações claras sobre um tema ou problema (Andrade, et al 2017).

Ainda com Andrade, et al. (2017) partir desse questionamento, procedeu-se às demais etapas para realização da revisão: formulação dos critérios de inclusão, definição das informações a serem extraídas das pesquisas selecionadas, avaliação rigorosa dos estudos incluídos na revisão integrativa, interpretação dos resultados e síntese do conhecimento.

Para a seleção dos estudos, foram pesquisadas as bases de dados: Medical Literature Analysis and Retrieval System Online (MEDLINE), bases Literatura Latino- Americana e do Caribe em Ciências da Saúde (LILACS), Scopus Info Site (SCOPUS), Scientific Electronic Library Online (SciELO), Bases de Dados em Enfermagem (BDENF) e Base de Datos Bibliográfica de La Fundación Index (CUIDEN), no período de Janeiro e Julho de 2021. As bases de dados foram escolhidas pela sua grande abrangência de estudos.

Realizou-se a seleção dos descritores após consulta ao DeCS e MeSH. Os descritores/palavras chaves utilizados foram: Incontinência Urinária $A N D$ Enfermagem $A N D$ Qualidade de vida; Enfermagem $A N D$ Assoalho pélvico $A N D$ fortalecimento de assoalho pélvico. Incontinência Urinária $A N D$ Enfermagem $A N D$ fortalecimento de assoalho pélvico. Obedeceu-se a mesma 
sequência na inserção dos descritores para as buscas, e diante do número reduzido de publicações, ampliou-se o estudo retrospectivo há 23 anos, delimitando-se entre 1998 e 2021 o período dos estudos, com o objetivo de abranger o maior quantitativo de publicações a respeito da temática, visto que não há muito sobre o tema relacionado à enfermagem. Os artigos selecionados atenderam aos seguintes critérios de inclusão: pesquisas disponíveis eletronicamente nas bases de dados selecionados, publicadas em periódicos revisados por pares, com amostra de mulheres climatéricas, textos completos disponíveis nos idiomas português, inglês e espanhol.

A busca nas bases de dados e no portal com a combinação dos descritores citados resultou em 09 artigos compatíveis com o estudo.

A leitura seletiva dos artigos foi realizada, inicialmente, com análise do título e resumo. Os artigos duplicados foram registrados apenas uma vez. Para garantir o registro conjunto de informações relevantes ao tema, foi utilizado instrumento próprio para análise dos artigos, o qual incluía dados de identificação (período publicado, idioma, país), aspectos metodológicos dos estudos (delineamento da pesquisa, amostragem e tratamento dos dados) e principais resultados e conclusões.

Na Figura 1, é possível analisar o processo de seleção nas bases de dados: Medical Literature Analysisand Retrieval System Online (MEDLINE), bases Literatura Latino- Americana e do Caribe em Ciências da Saúde (LILACS), Scopus Info Site (SCOPUS), Scientific Electronic Library Online (SciELO), Bases de Dados em Enfermagem (BDENF), e Base de Datos Bibliográfica de La Fundación Index (CUIDEN).

Figura 1. Processo de seleção dos estudos nas bases. Recife, Pernambuco, 2021.

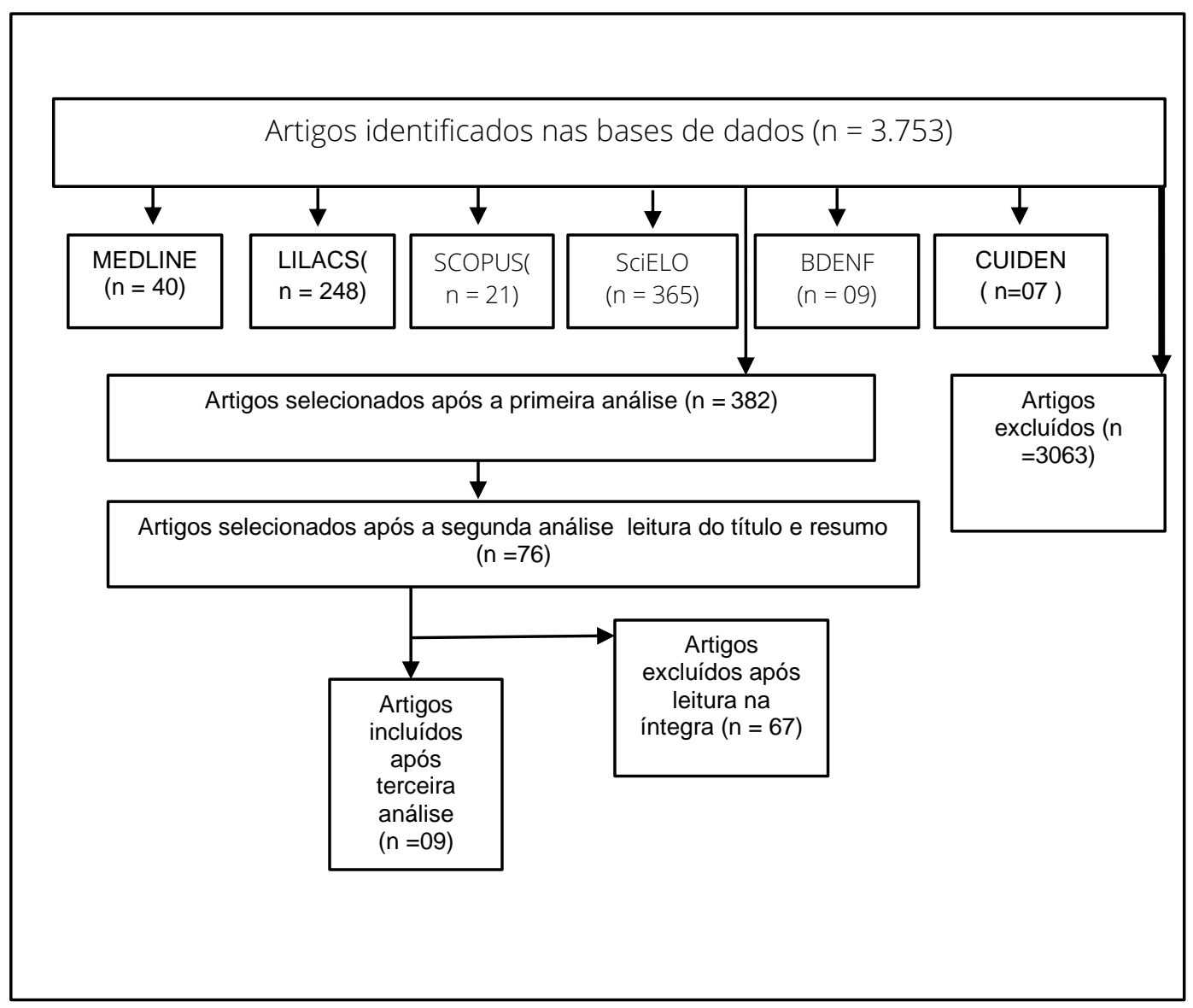

Fonte: Andrade et al. (2022). 


\section{Resultados e Discussão}

A amostra final desta revisão foi formada por vinte nove artigos disponíveis na íntegra em Português, Inglês e Espanhol, datados entre os anos de 1998 e 2021. Na seleção dos materiais utilizados encontram-se os resultados obtidos na caracterização da produção científica sobre as formas de avaliação da força da musculatura do assoalho pélvico e técnicas de fortalecimento deste, em mulheres com IU.

Desse modo, ficou evidenciado a escassez de trabalhos sobre o tema proposto relacionados à enfermagem, sendo necessário fazer uma análise dos últimos 20 anos para abranger o raio de busca. Nesse contexto, a abordagem e o detalhamento dos artigos indispensáveis neste estudo são demonstrados no Quadro 1.

Quadro 1. Caracterização de produção científica. Recife, Pernambuco, Brasil, 2022.

\begin{tabular}{|c|c|c|c|c|}
\hline Título do estudo & Ano/País & Delineamento/ Amostra & Resultados & Conclusão \\
\hline $\begin{array}{c}\text { A cinesioterapia como } \\
\text { tratamento da Incontinência } \\
\text { urinária na unidade básica } \\
\text { de saúde }\end{array}$ & 2005, Brasil. & $\begin{array}{l}40 \text { Mulheres, climatéricas, } \\
\text { que apresentavam } \\
\text { incontinência urinária de } \\
\text { esforço. }\end{array}$ & $\begin{array}{l}\text { Os resultados mostraram uma } \\
\text { diferença entre os dois } \\
\text { grupos, em que o grupo } \\
\text { experimental } 1 \text { - } 85 \%(\mathrm{n}=17) \\
\text { obteve melhora do quadro } \\
\text { urológico, já no grupo } \\
\text { experimental } 2 \text {-90\% }(\mathrm{n}=18) \\
\text { sem modificação. }\end{array}$ & $\begin{array}{c}\text { Exercícios associados a um } \\
\text { Programa de educação em saúde } \\
\text { pode ser uma alternativa eficaz, } \\
\text { menos onerosa e menos invasiva } \\
\text { em IU na UBS. }\end{array}$ \\
\hline $\begin{array}{c}\text { Avaliação da força muscular } \\
\text { do assoalho pélvico em } \\
\text { idosas com Incontinência } \\
\text { urinária }\end{array}$ & 2011, Brasil. & $\begin{array}{c}22 \text { mulheres submetidas à } \\
\text { anamnese, avaliação } \\
\text { funcional do assoalho } \\
\text { pélvico, quantificação da } \\
\text { Contração por meio da } \\
\text { palpação bidigital e } \\
\text { perineômetro, e avaliação } \\
\text { da QV por meio do (KHQ). }\end{array}$ & $\begin{array}{l}\text { Em relação ao grau de força } \\
\text { muscular foi observada } \\
\text { melhora significativa após o } \\
\text { tratamento }(\mathrm{p} \leq 0,001) \text { e } \\
\text { melhora do pico de pressão e } \\
\text { do tempo de contração } \\
\text { mensurados pelo } \\
\text { perineômetro. }\end{array}$ & $\begin{array}{c}\text { O protocolo Cinesioterapêutico } \\
\text { adotado foi eficaz para o FMAP e } \\
\text { para a melhora QV em idosas } \\
\text { incontinentes. }\end{array}$ \\
\hline $\begin{array}{l}\text { Avaliação da força muscular } \\
\text { do assoalho pélvico em } \\
\text { mulheres praticantes de Mat } \\
\text { Pilates. }\end{array}$ & 2014, Brasil. & $\begin{array}{c}\text { Estudo de caráter } \\
\text { interventivo, observacional } \\
\text { e descritivo, realizado entre } \\
\text { março a maio de } 2011 \text {, com } \\
\text { seis mulheres entre } 35 \text { e } 65 \\
\text { anos, através de aulas de } \\
\text { Mat Pilates, 2x semana. }\end{array}$ & $\begin{array}{c}\text { Observou-se que após } 8 \text { aulas } \\
\text { todas as voluntárias } \\
\text { ganharam força muscular nos } \\
\text { dois tipos de fibras, sendo } \\
\text { que } 84 \%(\mathrm{p}<0,05) \text { delas } \\
\text { tiveram maior ganho nas } \\
\text { fibras do tipo } 2 \text {. }\end{array}$ & $\begin{array}{c}\text { O Mat Pilates influencia no } \\
\text { aumento da força muscular do } \\
\text { assoalho pélvico, Na prevenção do } \\
\text { aparecimento de disfunções desta } \\
\text { musculatura. }\end{array}$ \\
\hline $\begin{array}{c}\text { Exercícios de Kegel } \\
\text { associados ao uso de cones } \\
\text { vaginais no Tratamento da } \\
\text { incontinência urinária: } \\
\text { estudo de caso }\end{array}$ & 2011, Brasil. & Estudo de caso & $\begin{array}{l}\text { Os exercícios de Kegel } \\
\text { associados ao uso dos cones } \\
\text { vaginais. A paciente referiu } \\
\text { estar sentindo os resultados } \\
\text { positivos após } 10 \text { sessões. }\end{array}$ & $\begin{array}{l}\text { Concluiu-se que os exercícios de } \\
\text { Kegel associados ao uso dos cones } \\
\text { vaginais, nesta paciente, levaram à } \\
\text { melhora da IU em um curto período } \\
\text { de tratamento. }\end{array}$ \\
\hline $\begin{array}{l}\text { Incontinência urinária em } \\
\text { mulheres no climatério: } \\
\text { efeitos Dos exercícios de } \\
\text { kegel }\end{array}$ & 2010, Brasil. & $\begin{array}{c}\text { Exercícios perineal em } \\
\text { mulheres entre } 45 \text { e } 60 \text { anos } \\
\text { de idade, na fase do } \\
\text { climatério, com } \\
\text { sintomatologia IU, } 08 \\
\text { pacientes, que realizaram } \\
10 \text { sessões de } 40 \text { ', 3x sem. } \\
\text { durante } 21 \text { dias. } \\
\end{array}$ & $\begin{array}{l}\text { Ao Final das intervenções, } \\
\text { constatou-se que não houve } \\
\text { solução da disfunção, porém } \\
\text { houve melhora significativa, } \\
\text { a maioria das passou da perda } \\
\text { de urina em jato para perda } \\
\text { em gotejamento. }\end{array}$ & $\begin{array}{l}\text { Concluiu-se que } 10 \text { sessões são } \\
\text { insuficientes para recuperação do } \\
\text { Tônus muscular pélvico e do } \\
\text { automatismo da contração do } \\
\text { assoalho Pélvico. }\end{array}$ \\
\hline $\begin{array}{l}\text { Influência dos exercícios } \\
\text { perineais e dos cones } \\
\text { vaginais, associados à } \\
\text { Correção postural, no } \\
\text { tratamento da incontinência } \\
\text { urinária feminina }\end{array}$ & 2006, Brasil. & $\begin{array}{c}\text { Foram avaliadas } 12 \\
\text { mulheres com IU, com } \\
\text { idade média de } 52,3 \pm 9,7 \text {, } \\
\text { em } 10 \text { sessões } \\
\text { fisioterapêuticas, } 2 \text { x sem., } \\
\text { em } 2 \text { grupos: grupo } \text { a: } \mathrm{n}= \\
6 \text {, utilizando exercícios } \\
\text { perineais e grupo } b: \mathrm{n}=6 \text {, } \\
\text { utilizando cones Vaginais. }\end{array}$ & $\begin{array}{c}\text { Na comparação dos Dados } \\
\text { pré e pós-intervenção, foi } \\
\text { observado, para ambos os } \\
\text { grupos, diminuição } \\
\text { estatisticamente significativa } \\
\text { da perda urinária (p } \leq 0,05) \text {, } \\
\text { ganho de contração muscular } \\
\text { do assoalho pélvico e } \\
\text { diminuição significativa da } \\
\text { sensação de umidade. }\end{array}$ & $\begin{array}{c}\text { A correção estática da pelve, } \\
\text { através dos exercícios posturais, } \\
\text { constitui uma modalidade efetiva de } \\
\text { intervenção na pele feminina, } \\
\text { Quando associada à e educação } \\
\text { perineal. }\end{array}$ \\
\hline $\begin{array}{c}\mathrm{O} \text { fortalecimento do } \\
\text { assoalho pélvico com cones } \\
\text { vaginais: programa de } \\
\text { atendimento domiciliar. }\end{array}$ & 2009, Brasil. & $\begin{array}{l}\text { Estudo de caso. Foi } \\
\text { realizado através de um } \\
\text { protocolo de exercícios } \\
\text { com cones vaginais pó } 8 \\
\text { sem., 3x sem., 2x dia. }\end{array}$ & $\begin{array}{l}\text { Executou treinamento pélvico } \\
\text { com cones vaginais durante } 8 \\
\text { semanas com bons resultados. }\end{array}$ & $\begin{array}{l}\text { O programa de exercícios com } \\
\text { cones vaginais a domicílio mostrou- } \\
\text { se efetivo na resolução da IUE. }\end{array}$ \\
\hline
\end{tabular}




\begin{tabular}{|c|c|c|c|c|}
\hline $\begin{array}{l}\text { Programa de reabilitação do } \\
\text { assoalho pélvico: relato de } \\
10 \text { anos de experiência }\end{array}$ & 2017, Brasil. & $\begin{array}{c}\text { Relatar a criação, } \\
\text { experiência de implantação } \\
\text { e atendimento realizado no } \\
\text { Programa de Reabilitação } \\
\text { do Assoalho Pélvico } \\
\text { (PRAP), desenvolvido em } \\
\text { um centro de saúde de } \\
\text { Campinas, SP, Brasil. }\end{array}$ & $\begin{array}{l}\text { Atendido } 102 \text { pacientes com } \\
\text { IU e outras disfunções do } \\
\text { assoalho pélvico e do trato } \\
\text { urinário inferior, formado } 480 \\
\text { alunos, capacitado oito } \\
\text { profissionais de saúde e } \\
\text { estimulado pesquisas. }\end{array}$ & $\begin{array}{l}\text { As atividades preventivas e de } \\
\text { reabilitação do assoalho pélvico } \\
\text { constituem-se áreas de importante } \\
\text { atuação do enfermeiro e iniciativas } \\
\text { como a relatada contribuem para a } \\
\text { formação profissional e prática } \\
\text { baseada em evidências. }\end{array}$ \\
\hline $\begin{array}{c}\text { Terapia comportamental } \\
\text { para incontinência urinária } \\
\text { da mulher idosa: uma ação } \\
\text { do enfermeiro }\end{array}$ & 2010, Brasil. & $\begin{array}{l}\text { Trata-se da descrição dos } \\
\text { resultados de uma } \\
\text { experiência, a partir de } \\
\text { dados coletados nos } \\
\text { prontuários de mulheres } \\
\text { atendidas no ambulatório } \\
\text { geriátrico de um hospital } \\
\text { universitário. } 12 \text { pacientes. }\end{array}$ & $\begin{array}{l}08 \text { pacientes referiram que os } \\
\text { exercícios perineais foram o } \\
\text { elemento da terapia que mais } \\
\text { ajudou. } 06 \text { referiram que a } \\
\text { TC melhorou a auto-estima e } \\
\text { o autocuidado, ajudou a lidar } \\
\text { com o problema. Além disso, } \\
03 \text { que a TC minimizou os } \\
\text { sintomas. }\end{array}$ & $\begin{array}{l}\text { Os resultados apontam para o valor } \\
\text { desta ação de enfermagem para a } \\
\text { prática clínica. A TC deve ser } \\
\text { aplicada antes de encaminhar uma } \\
\text { paciente com IU para realizar um } \\
\text { procedimento cirúrgico, sugerimos } \\
\text { a padronização das ações de } \\
\text { enfermagem incluídas neste } \\
\text { procedimento. }\end{array}$ \\
\hline
\end{tabular}

Fonte: Andrade et al., (2022).

\section{Fortalecimento dos músculos do assoalho pélvico: cuidados de enfermagem e qualidade de vida}

Por volta dos anos 1950, Arnold Kegel, médico ginecologista, foi o primeiro a introduzir o treinamento da musculatura do assoalho pélvico feminino para tratar a IU (Ramos \& Oliveira, 2010, Gonçales, et al., 2012). Neste período, muitos fisioterapeutas do Reino Unido estiveram empenhados no tratamento da IU, usando os exercícios para o assoalho pélvico (cinesioterapia) e, a eletroestimulação vaginal (Sousa, et al., 2011). Estudo relatou índice de cura de $84 \%$ e melhora não só na continência urinária, mas também no prazer sexual (Berquó, et al., 2013).

A assistência de enfermagem em ginecologia e sexualidade humana se baseia em oferecer consulta de enfermagem, com orientação de exercícios baseados nestes exercícios (Higa, et al., 2008). A terapêutica é conservadora e é realizada através de técnicas que visam o fortalecimento da musculatura do assoalho pélvico, uma vez que a disfunção muscular perineal representa importante fator etiopatogênico (Netter, 2015).

De acordo com Arruda, et al., (2007) e Caldas, et al., (2010), a abordagem visa a uma rearmonização para correção da estática pélvica e um fortalecimento dos componentes esfincterianos, para um aumento do tônus e uma correta transmissão das pressões intra-abdominais, que refletirão no mecanismo da continência, tendo em vista que uma má postura da pelve pode influenciar a funcionalidade dos músculos do assoalho pélvico e refletir nesse conjunto de sustentação. Baseiam-se na contração voluntária dos músculos perineais para reeducar o assoalho pélvico e aumentar seu tônus muscular (Herrmann, et al., 2003).

Marques \& Freitas (2005) trazem seus relatos que o tratamento depende das condições de saúde da pessoa, do tipo e estágio da incontinência e pode ser tanto cirúrgico quanto conservador com medicamentos, exercícios ou terapia comportamental. No estudo realizado por Caldas, et al., (2010), com idosas com incontinência urinária, onde foi utilizada a Terapia Comportamental, o Processo de Enfermagem foi utilizado, envolvendo o planejamento de estratégias de intervenção, sua aplicação e avaliação dos resultados. Neste estudo, as pacientes foram orientadas na consulta de Enfermagem quanto aos exercícios perineais, ou de Kegel, que melhora a musculatura do assoalho pélvico, assim como cuidados básicos de higiene, hidratação, prevenção de lesões no períneo, entre outros (Caldas, et al., 2010).

Matheus, et al., (2002) relata que as publicações realizadas por Enfermeiros neste campo são todas descrevendo ações de enfermagem e orientações nas consultas de enfermagem, de modo a melhorar a QV da população de uma forma geral, com informações fáceis, baratas e pouco invasivas, ou seja, contemplando a Lei do Exercício Profissional de Enfermagem e o Processo de Enfermagem conforme Resolução COFEN 358/2009.

Considerando o que consta no Decreto 94.406/87 que regulamenta a Lei 7.498/86 Lei do Exercício Profissional de Enfermagem, o enfermeiro pode e deve exercer o atendimento visto que não é nada invasivo. 
Frente ao exposto, conclui-se que a atuação do Enfermeiro na reabilitação do trato urinário inferior faz parte da ação do Enfermeiro dentro do Processo de Enfermagem, assegurada pela Lei do Exercício Profissional de Enfermagem, por meio da consulta de enfermagem e utilizando orientações e métodos não invasivos. A assistência de enfermagem em Uroginecologia, engloba atenção urológica, ginecológica e a sexualidade humana, e baseia-se em oferecer consulta de enfermagem através do processo de enfermagem com a sequência de passos que ordenam a consulta (Lopes, et al., 2017; Gouveia, et al., 2013).

Além de sistematizar o processo de enfermagem nesta área, validaram roteiro de consulta, e afirmam que não é um atendimento que deve se restringir apenas às enfermeiras estomaterapeutas, mas a sequência de passos para atender pessoas com queixas de IU, é pertinente às enfermeiras da urologia, ginecologia ou obstetrícia que podem fazer esse atendimento desde que sejam capacitadas e/ou tenham recebido treinamento adequado (Lopes, et al., 2017; Lucas, et al., 2012).

A orientação sobre o autocuidado é uma das atribuições do enfermeiro sendo assim, a conscientização da existência da musculatura perineal, da necessidade do seu fortalecimento para garantir saúde uroginecológica e sexual, são parte da assistência prestada na referida consulta (Herrmann, et al., 2003).

Esta abordagem visa reestabelecer a harmonia por correção da estática pélvica e proporcionar um fortalecimento dos esfíncteres, aumentando do tônus e proporcionando uma correta transmissão das pressões intra-abdominais, desencadeando a continência, a desarmonização da postura da pelve influencia a funcionalidade dos músculos do assoalho pélvico e reflete na capacidade de sustentação, perdendo sua capacidade funcional (Morales, 2012). Por isso os exercícios baseiam-se na contração voluntária dos músculos perineais para reeducar o assoalho pélvico e aumentar seu tônus muscular (Silva \& Oliva, 2011).

Segundo a OMS a QV refere-se à percepção do indivíduo, de sua vida, sua cultura e valores pessoais, suas perspectivas e suas expectativas pessoais e sociais. Deste modo, QV é uma consideração ampla entre aspectos físicos, psicológicos, nível de independência, relações sociais e crenças pessoais (Dreher, et al., 2009).

Ou seja, para uma melhor QV, é importante a prevenção de IU, diagnóstico e tratamento precoce. O que exige uma avaliação precoce, uso de estratégias de intervenção e análise da resposta aos exercícios de FMAP sob um olhar biopsicossocial, conforme preconiza a OMS, visando a manutenção das atividades rotineiras e participação social (Dreher, et al., 2009).

Frente ao exposto, conclui-se que a reabilitação do trato urinário inferior faz parte do Exercício Profissional de Enfermagem, por meio da consulta de enfermagem em todas as suas etapas e a IU deve ser tratada por ter influência direta na QV, principalmente das mulheres climatéricas.

\section{Conclusão}

A atual revisão demonstrou que as publicações realizadas nesta área, e principalmente por Enfermeiros, ainda tem pouca representatividade, e que devido à relevância do tema precisamos desenvolver outros estudos evidenciando a relevância do trabalho do enfermeiro, no ato da consulta de enfermagem e seu amparo legal, avaliando a força do assoalho pélvico, e conforme a detecção do enfraquecimento muscular iniciar a orientação supervisionada dos exercícios e as devidas orientações nas consultas e em grupos focais, de modo a melhorar a QV da população feminina de uma forma geral, com informações fáceis, que transmitem segurança às mulheres e incentivam este tratamento não invasivo e que permite manter a rotina e convívio social.

Ressaltamos que a avaliação da função dos músculos do assoalho pélvico através da palpação bidigital e observação dos MAP, podendo ser associado a perineometria são as formas as mais efetivas, e que são de fácil treinamento, ou seja, acessível ao enfermeiro, e isto trás muitos benefícios enquanto saúde pública. Sendo o acesso mais fácil, rápido e cômodo da usuária ao sistema, tendo um tratamento e acompanhamento de resultados de baixo custo com qualidade, além de diminuir as chances de tratamento cirúrgicos, de alto custo, de risco maior à vida e de congestionamento do sistema terciário de atenção à saúde. 
Quanto às formas de FMAP através dos exercícios, a literatura demonstra em todos os estudos acessados melhora significativa na QV das mulheres incontinentes, com cura da IU em $80 \%$ dos casos, sendo até, maior em algumas amostras. A base da orientação de enfermagem é através da conscientização da necessidade do autocuidado, por meio da reeducação Pélvica Perineal, que através do processo de enfermagem durante a consulta em ginecologia/ uroginecologia, e através da orientação dos exercícios, por um período maior que 21 sessões apontam ser uma técnica assertiva que deve ser utilizada para o FMAP, pela relevância à prevenção e controle da IU, durante todas as fases vida.

E esta consulta de enfermagem em ginecologia/uroginecologia com o treinamento dos enfermeiros pode e deve acontecer em qualquer território, visto que a proximidade à unidade de saúde favorece a adesão ao tratamento individual e em grupo. O que possibilitará a integralidade na atenção à saúde da mulher, seguindo os princípios do Sistema Único de Saúde.

\section{Referências}

Andrade, S. R. et al. (2017). O estudo de caso como método de pesquisa em enfermagem: uma revisão integrativa. Texto \& contexto enferm. $26(4)$, e5360016.

Arruda, R. M. et al. (2007). Hiperatividade do detrusor: comparação entre oxibutinina, eletroestimulação funcional do assoalho pélvico e exercícios perineais. Estudo randomizado. Rev. Bras. Ginecol. Obstet. 29 (9).

Berquó, M. S. et al. (2013). Fisioterapia no tratamento da urgência miccional feminina. Rev. FEMINA. 41(2): 108-112.

Bertoldi, J. T. et al. (2014). Fisioterapia na incontinência urinária de esforço: revisão de literatura. Cinergis; 15(4): $224-229$.

Botelho F. et al. (2007). Incontinência urinária feminina. Acta Urológica. 24(1): 79-82.

Brasil. Ministério da Saúde. (2013). Saúde sexual e reprodutiva. Caderno de Atenção Básica. Brasília - DF. https://bvsms.saude.gov.br/bvs/publicacoes/sal de_sexual_saude_reprodutiva.pdf

Brasil. Ministério da Saúde. (2008). Manual de atenção à mulher no climatério/menopausa. https://bvsms.saude.gov.br/bvs/publi cacoes/manual_atencao_mulher_climaterio.pdf

Brasil. Ministério da Saúde. (2016). Protocolos da atenção básica: saúde das mulheres. https://bvsms.saude.gov.br/bvs/publicacoes/p rotocolos_atencao_basica_saude_mulheres.pdf

Caldas, C. P. et al. (2010). Terapia comportamental para incontinência urinária da mulher idosa: uma ação do enfermeiro. Texto Contexto Enferm, 19(4): 783-8. COFEN. Conselho Federal de Enfermagem. (2016). Parecer de câmara técnica no 04/2016/CTAS/COFEN. http://www.cofen.gov.br/parecer-no042016ctascofen_45837.html

Dreher, D. Z. et al. (2009). O fortalecimento do assoalho pélvico com cones vaginais: programa de atendimento domiciliar. Scientia Medica., 19(1): 43-49.

Ferreira M., SANTOS, P. (2009). Princípios da Fisiologia do Exercício no Treino dos Músculos do Pavimento Pélvico. Acta urológica., 26(3): 31-38.

Gonçales, A. T. et al. (2012). Avaliação da qualidade de vida após o uso da eletroestimulação associada a exercícios perineais de kegel no tratamento de incontinência urinária por esforço relato de caso. Revista Hórus, 7(2): 41-23.

Guarisi, T. et al. (2001). Incontinência urinária entre mulheres climatéricas brasileiras: inquérito domiciliar. Rev. Saúde Pública, 35(5): 428-435.

Gouveia, P. F. et al. (2013). Métodos de avaliação do assoalho pélvico. Moreira Jr. Editor, 70(6): 232-238.

Herrmann, V. et al. (2003). Eletroestimulação transvaginal do assoalho pélvico no tratamento da incontinência urinária de esforço: avaliações clínicas e ultrassonográficas. Rev. Assoc. Med., 49(4): 401-405.

Higa, R. et al. (2008). Fatores de risco para incontinência urinária na mulher. Rev. esc. enferm. USP, 42(1): 187-192.

Lopes, M. H. B. M. et al. (2017). Programa de reabilitação do assoalho pélvico: relato de 10 anos de experiência. Rev. Bras. Enferm., 70(1): 219-23.

Lucas, M. G. et al. (2012). diretrizes para incontinência urinária. Tradução da European Association of Urology - EAU, do "Guidelines Office”. https://uroweb.org/wp-content/uploads/Urinary-Incontinence-2012-pocket-1.pdf

Marques, K. S. F., Freitas, P. A. C. (2005). A cinesioterapia como tratamento da incontinência urinária na unidade básica de saúde. Fisioterapia em Movimento, 18(4): 63-67.

Matheus, L. M. et al. (2002). Influência dos exercícios perineais e dos cones vaginais, associados à correção postural, no tratamento da incontinência urinária feminina. Rev. bras. fisioter., 10(4): 387-392.

Morales, M. M. (2012). Incontinencia urinaria Femenina. Revista medica de costa rica y centroamerica, (602) 225-230.

Mourão, L. F. et al. (2017). Caracterização e Fatores de Risco de Incontinência Urinária em Mulheres Atendidas em uma Clínica Ginecológica. ESTIMA, 15(2): 82-91. 
Research, Society and Development, v. 11, n.3, e31611326513, 2022

(CC BY 4.0) | ISSN 2525-3409 | DOI: http://dx.doi.org/10.33448/rsd-v11i3.26513

Netter, F. H. (2015). Atlas de anatomia humana. 6. ed. Brasil: Rio de Janeiro: Elsevier; 624p.

Oliveira, L. G. P. et al. (2018). Incontinência urinária: a atuação do profissional de enfermagem. Revista Eletrônica Acervo Saúde, (18), e118. https://doi.org/10.25248/reas.e118.2019

Paris. (2008). International Continence Society. Recommendations of the International Scientific Committee: evaluation and treatment of urinary incontinence, Pelvic Organ Prolapse and Faecal Incontinence. $4^{\mathrm{a}}$ International Consultation on Incontinence.

Ramos, A. L., Oliveira, A. A. C. (2010). Incontinência urinária em mulheres no climatério: efeitos dos exercícios de kegel. Revista Hórus, 4(2): 264-275.

Santos Menezes, C. N. (2021). Os benefícios da fisioterapia pélvica na melhora da libido no período pós-parto. Revista Cathedral, 3(2), 56-65.

Silva, A. M. N., Oliva, L. M. P. (2011). Exercícios de Kegel associados ao uso de cones vaginais no tratamento da incontinência urinária: estudo de caso. Scientia Medica, 21(4): 264-275.

Sousa, C. B., Souza, V. S., \& Figueredo, R. C. (2020). Disfunções sexuais femininas: recursos fisioterapêuticos na anorgasmia feminina pela fraqueza do assoalho pélvico. Multidebates, 4(2), 176-188.

Sousa, J. G. et al. (2011). Avaliação da força muscular do assoalho pélvico em idosas com incontinência urinária. Fisioter Mov., 24(1): 39-46.

Souza, M. T. et al. (2010). Revisão integrativa: o que é e como fazer. Einstein, 8(1):102-106.

SOBRAC. (2003). Sociedade Brasileira de Climatério. Consenso brasileiro multidisciplinar de assistência à mulher climatérica [http://pesquisa.bvsalud.org/portal/resource/ pt/lis-LISBR1.1-21461 